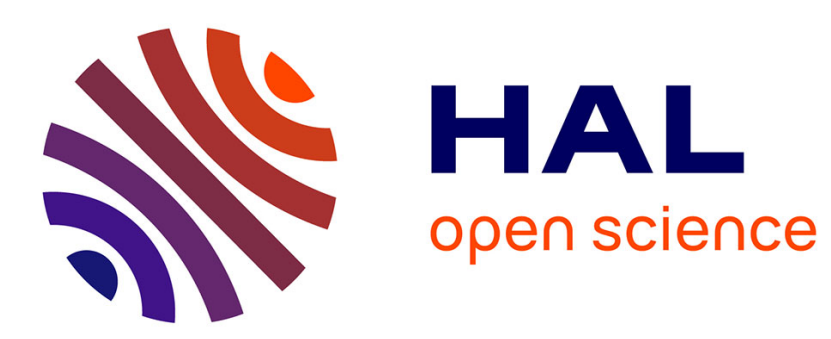

\title{
Insight et comportements violents chez les patients souffrant d'une schizophrénie
}

M. Voyer, N. Jaafari, J.-L. Senon

\section{To cite this version:}

M. Voyer, N. Jaafari, J.-L. Senon. Insight et comportements violents chez les patients souffrant d'une schizophrénie. Annales Médico-Psychologiques, Revue Psychiatrique, 2011, 169 (7), pp.441. 10.1016/j.amp.2011.06.006 . hal-00789302

\section{HAL Id: hal-00789302 \\ https://hal.science/hal-00789302}

Submitted on 18 Feb 2013

HAL is a multi-disciplinary open access archive for the deposit and dissemination of scientific research documents, whether they are published or not. The documents may come from teaching and research institutions in France or abroad, or from public or private research centers.
L'archive ouverte pluridisciplinaire HAL, est destinée au dépôt et à la diffusion de documents scientifiques de niveau recherche, publiés ou non, émanant des établissements d'enseignement et de recherche français ou étrangers, des laboratoires publics ou privés. 


\section{Accepted Manuscript}

Title: Insight et comportements violents chez les patients souffrant d'une schizophrénie

Authors: M. Voyer, N. Jaafari, J.-L. Senon

PII: $\quad$ S0003-4487(11)00165-X

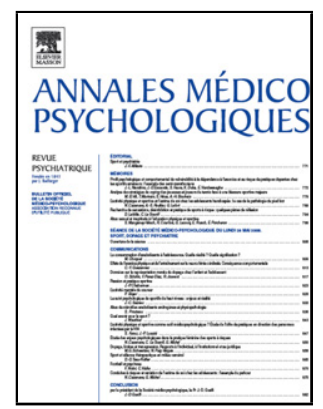

DOI: $\quad$ doi:10.1016/j.amp.2011.06.006

Reference: $\quad$ AMEPSY 1347

To appear in: $\quad$ Annales Médico-Psychologiques

Please cite this article as: Voyer M, Jaafari N, Senon J-L, Insight et comportements violents chez les patients souffrant d'une schizophrénie, Annales medio-psychologiques (2010), doi:10.1016/j.amp.2011.06.006

This is a PDF file of an unedited manuscript that has been accepted for publication. As a service to our customers we are providing this early version of the manuscript. The manuscript will undergo copyediting, typesetting, and review of the resulting proof before it is published in its final form. Please note that during the production process errors may be discovered which could affect the content, and all legal disclaimers that apply to the journal pertain. 


\title{
Communication
}

\section{Insight et comportements violents chez les patients souffrant d'une schizophrénie} Insight and violent behaviour in patients with schizophrenia

\author{
M. Voyer $^{\text {a }}$, N. Jaafari ${ }^{\text {a }}$, J.-L. Senon ${ }^{\text {a }}$ \\ ${ }^{a}$ Unité de recherche clinique intersectorielle en psychiatrie $d u$ \\ Centre Hospitalier Henri Laborit, Poitiers, France
}

Auteur correspondant: Dr Mélanie Voyer, Pavillon Toulouse, BP 587, Centre Hospitalier Henri Laborit, 86021 Poitiers, France

Adresse email : mel.voyer@wanadoo.fr

\section{Résumé}

La schizophrénie est associée, dans la croyance populaire, à la violence et à la dangerosité. Les études réalisées ces dernières années ont retrouvé une augmentation du risque de violence chez les sujets souffrant de troubles mentaux, mais cette augmentation serait plurifactorielle. La capacité d’insight des sujets souffrant de schizophrénie a été étudiée pour sa participation possible à l'augmentation du risque de violence, mais les résultats sont, à ce jour, contradictoires, ne permettant pas d'en tirer des conclusions.

Mots clés : Agression ; Dangerosité ; Insight ; Schizophrénie ; Violence

\begin{abstract}
Schizophrenia is associated in popular belief with violent behaviour. In recent years, many studies conducted on this topic have found an increased risk of violence in subjects with mental disorders. However, violent behaviour in patients with schizophrenia is also multifactorial. Some studies report that risk of violent behaviour increased in patients with schizophrenia with poor insight, but others did not confirm this idea. The picture is not clear, and prospective studies should be conducted on this topic.
\end{abstract}

Keywords: Aggression; Dangerosity; Insight; Schizophrenia; Violence 
La violence fait partie intégrante de la psychiatrie, que ce soit au travers d'actes autoagressifs, qui sont les plus fréquents, mais parfois aussi au travers d'actes de violence dirigés vers autrui. C'est pourtant cette violence hétéro-agressive qui est mise le plus souvent en avant par les médias et les pouvoirs publics, renvoyant à la population l'image du « schizophrène dangereux évadé de l’asile ». La schizophrénie est associée, dans l’imaginaire collectif, à la dangerosité, l'agressivité, la violence, avec une stigmatisation importante des individus présentant un trouble psychotique. C’est pour tenter de diminuer cet « étiquetage » des patients souffrant de schizophrénie que des études ont été menées visant à rechercher le lien réel entre maladie mentale et violence.

Les études réalisées ces 20 dernières années ont montré une augmentation du risque de violence hétéro-agressive chez les sujets souffrant de schizophrénie, risque deux à trois fois supérieur à celui de la population générale [5,8,10]. Mais même s’il existe une augmentation du risque de comportement violent en cas de trouble mental et de schizophrénie, il est néanmoins important de souligner que la plupart des actes violents graves, comme les homicides, sont commis par des individus ne présentant pas de trouble psychotique (environ $10 \%$ des auteurs d'homicide souffrent d'un trouble psychotique [13]). Si les études montrent une influence importante de l'alcool ou des substances psychoactives dans l'augmentation du risque de violence chez des individus souffrant de schizophrénie [8,11], d'autres facteurs comme les antécédents de violence [8], les symptômes psychotiques positifs [16], l'association à un trouble de la personnalité antisociale ou l'existence de traits psychopathiques, la mauvaise observance du traitement et la non-adhésion aux soins [9] semblent participer à cette augmentation.

\section{1. Études sur le lien entre insight et violence}

Peu d'études ont été réalisées sur le lien entre l’insight et le risque de violence chez les sujets souffrant d'un trouble schizophrénique. La première étude prospective a été réalisée par Arango et al. (1999) [2] dans une unité d'admission d'un hôpital psychiatrique, à partir d'une population de 63 individus souffrant de schizophrénie ou de trouble schizo-affectif. Les actes agressifs commis pendant l'hospitalisation ont été évalués à l'aide de l'Overt Aggression Scale (OAS). L'insight a été évalué à l'aide de la Scale to assess Unawareness in Mental Disorder (SUMD). Seize patients ont été violents contre autrui, tous dans la première semaine suivant l'admission. Les auteurs ont retrouvé que ces patients avaient significativement moins d’insight dans la reconnaissance des symptômes de leur maladie et concernant la nécessité du 
traitement, à l'admission et à la sortie de l'hôpital, par rapport aux patients non violents. Ils ont également mis en évidence que l’insight dans les symptômes psychotiques était le meilleur facteur prédictif de violence, avec une haute valeur prédictive positive et négative. Yen et al. (2002) [19] ont suivi, prospectivement, 74 patients souffrant de schizophrénie, en ambulatoire pendant un an. Ils ont évalué l'insight au début de l'étude avec la Schedule for the Assessment of Insight (SAI) et la Schedule for the Assessment of Insight Expanded-version (SAI-E), et la violence (violence hétéro-agressive contre les personnes ou les biens) avec la Violence and Suicide Assessment Scale (VASA) après le suivi d'un an. Onze des patients ont été violents. Les auteurs ne retrouvent aucune différence dans les scores d'insight entre les patients violents et les non-violents. Waldheter et al. (2005) [18], dans une étude prospective de suivi de 29 patients hospitalisés et présentant des troubles mentaux graves, ont évalué les capacités d'insight avec l'Insight Scale (IS [4]). Les actes de violence sont relevés à partir de la Modified Aggression Scale (MOAS) (agressions verbales, physiques contre les objets, physiques contre soi et contre autrui). Dans cette étude, l’insight n'est pas associé à la violence future chez les individus souffrant d'un trouble mental grave, mais il n'y a pas de résultats spécifiques pour les sujets présentant une schizophrénie. Dans une autre étude, Arango et al. (2006) [3] ont suivi pendant un an 46 patients souffrant de schizophrénie et ayant déjà été violents. Les auteurs n’ont pas retrouvé de différence d’insight (mesuré par la SUMD au début de l'étude) entre les patients ayant réitéré des actes de violence et ceux n’ayant pas récidivé (violence avec lésions physiques à la MOAS). Enfin, Lincoln et al. en 2008 [14], ont suivi en ambulatoire pendant deux ans après leur sortie de l’hôpital, 209 sujets souffrant de schizophrénie, dont 27 ont commis un acte violent (violence physique contre autrui, violence avec menace d'une arme ou violence sexuelle), la violence étant rapportée par le patient lui-même ou par un proche. L’insight était quant à lui évalué par la Positive and Negative Syndrome Scale (PANSS) et l'Historical, Clinical, Risk Management-20 (HCR-20). Ces auteurs n'ont pas retrouvé de lien entre le niveau d'insight et la survenue de comportements violents après contrôle des variables de psychopathie (score à la Hare's Psychopathy Checklist-Revised [PCL-R]) et d'intensité de la symptomatologie psychotique positive (PANSS).

Concernant les études rétrospectives, il existe également des différences dans les méthodes de mesure de l'insight et de la violence. Cheung et al. (1997) [7] ont étudié les capacités d'insight de 62 patients hospitalisés, souffrant de schizophrénie, à partir de l'item 12 de la PANSS. Trente et un d'entre eux avaient été violents contre autrui ou contre des biens, la violence étant mesurée par l’OAS, mais les auteurs n’ont pas relevé de relation significative 
entre l'insight et la violence. De même, Swartz et al. (1998) [17], en s'intéressant cette fois-ci à 331 patients hospitalisés souffrant de troubles mentaux sévères (trouble schizophrénique, trouble bipolaire et épisode dépressif majeur) et en recherchant les agressions ou menaces graves sur les dossiers médicaux ou bien par les entretiens avec le malade ou ses proches, n’ont pas retrouvé d'association entre l'insight (mesuré avec l'Insight and Treatement Attitude Questionnaire [ITAQ]) et la violence. Buckley et al. (2004) [6], en revanche, ont montré que les patients hospitalisés souffrant de troubles schizophréniques et ayant commis des infractions avec violence contre autrui ou contre des biens $(\mathrm{n}=115)$ avaient un plus faible insight que les patients sans antécédents d’infractions. L’insight était ici évalué grâce à la SUMD, l'item 12 de la PANSS, et l'Eisner Scale.

Foley et al. (2005) [12], en s’intéressant à 157 patients hospitalisés pour un premier épisode psychotique dont 52 violents, ont trouvé que la violence serait associée significativement à un manque d'insight (OR =2,97, 95 \% IC 1,03-8,56). L'insight était mesuré par l’item G12 de la PANSS et la violence par l'échelle OAS. En étudiant 60 patients violents, souffrant de troubles schizophréniques ou de troubles affectifs avec symptômes psychotiques et pris en charge dans une unité de psychiatrie médico-légale, Alia-Klein et al. (2007) [1] ont mis en évidence que le niveau d'insight (évalué avec la SUMD) était associé à la sévérité d'actes violents (évalués par la Violence Assessment Scale (VAS) et l’OAS), et ce indépendamment de l’observance thérapeutique. Enfin, Soyka et al. (2007) [15], dans une étude regroupant 1662 patients présentant une schizophrénie, dont 169 avaient commis des infractions avec violence (10\%), ont retrouvé que ceux qui avaient été violents avaient un insight plus faible à la sortie de l’hôpital que ceux qui ne l’avaient pas été.

La présentation de ces études met en évidence une hétérogénéité des résultats. Le lien entre l'insight et le risque de violence apparaît, à leur lecture, difficile à établir. Cinq études sur onze retrouvent un lien entre un faible insight et la violence, mais parmi ces cinq études, une seule est réellement prospective. L’insight est une capacité du sujet qui varie avec le temps (en fonction du traitement, de la prise en charge, de l'environnement du sujet), c'est une variable dynamique. Les études évaluant prospectivement la violence, à distance de celles de l'insight, ne retrouvent aucun lien entre ces deux variables, montrant ainsi une efficacité prédictive de l'insight plutôt sur la violence à court terme, comme dans l'étude d'Arango [2].

De plus, un certain nombre de variables semblent interagir avec l'insight (intensité des symptômes, addiction, trouble de la personnalité antisociale...) dans l'émergence de comportements violents, mais ces variables sont rarement prises en compte dans les études, ce 
qui peut également modifier les résultats concernant le lien entre insight et risque de violence. De plus, les violences prises en compte dans ces études (de la « simple » violence verbale aux violences ayant fait l'objet de poursuites pénales), mais également les populations (hospitalisés ou suivis en ambulatoire, médico-légaux...) sont disparates, rendant difficiles les comparaisons. Enfin, aucune étude ne prend en compte l'impact de la prise en charge thérapeutique qui peut modifier les résultats.

Du fait des nombreux biais mis en évidence, il est à l'heure actuelle impossible d'affirmer l'existence d'un lien entre un faible insight et une augmentation du risque de survenue d'actes de violence. Il serait intéressant, pour aller plus loin dans ces recherches, de développer des études prospectives prenant en compte uniquement les actes de violence hétéro-agressifs avec contact physique, dans une population suffisante pour pouvoir déterminer un lien statistiquement significatif. La capacité d'insight du patient devrait être évaluée à proximité de l'acte violent, à l'aide d'une échelle multidimensionnelle comme la SUMD. De plus, il serait important de pouvoir prendre en compte d'autres variables comme l'intensité des symptômes productifs, l'existence d'un trouble de la personnalité antisociale co-morbide, d'une addiction, qui sont des facteurs de risque de violence reconnus. Seule la mise en place de ce type d'étude permettrait peut-être de mettre en évidence le lien entre l’insight et la violence chez les sujets souffrant de schizophrénie.

\section{Conflit d'intérêt : à compléter par l'auteur}

\section{Références}

[1] Alia-Klein N, O'Rourke TM, Goldstein RZ, Malaspina D. Insight into illness and adherence to psychotropic medications are separately associated with violence severity in a forensic sample. Aggress Behav 2007;33:86-96.

[2] Arango C, Calcedo Barba A, Gonzalez S, Calcedo Ordonez A. Violence in inpatients with schizophrenia: a prospective study. Schizophr Bull 1999;25:493-503.

[3] Arango E, Bombin I, Gonzales T, Garcia I, Bobes J. Randomised clinical trial comparing oral versus depot formulations of zuclopenthixol in patients with schizophrenia and previous violence. Eur Psychiatry 2006;21:34-40.

[4] Birchwood M, Smith J, Drury V, Healy J, Macmillan F, Slade M. A self-report Insight Scale for psychosis: reliability, validity and sensitivity to change. Acta Psychiatr Scand 1994;89:62-7.

[5] Brennan PA, Mednick SA, Hodgins S. Major mental disorders and criminal violence in a 
Danish birth cohort. Arch Gen Psychiatry 2000;57:494-500.

[6] Buckley PF, Hrouda DR, Friedman L, Noffsinger SG, Resnick PJ, Camlin-Shingler K. Insight and its relationship to violent behavior in patients with schizophrenia. Am J Psychiatry 2004;161:1712-4.

[7] Cheung P, Schweitzer I, Crowley K, Tuckwell V. Violence in schizophrenia: role of hallucinations and delusions. Schizophr Res 1997;26:181-90.

[8] Elbogen EB, Johnson SC. The intricate link between violence and mental disorder: results from the National Epidemiologic Survey on Alcohol and Related Conditions. Arch Gen Psychiatry 2009;66:152-61.

[9] Erb M, Hodgins S, Freese R, Muller-Isberner R, Jockel D. Homicide and schizophrenia: maybe treatment does have a preventive effect. Crim Behav Ment Health 2001;11:6-26.

[10] Fazel S, Grann M, Carlstrom E, Lichtenstein P, Langstrom N. Risk factors for violent crime in Schizophrenia: a national cohort study of 13.806 patients. J Clin Psychiatry 2009;70:362-9.

[11] Fazel S, Langstrom N, Hjern A, Grann M, Lichtenstein P. Schizophrenia, substance abuse, and violent crime. JAMA 2009;301:2016-23.

[12] Foley SR, Kelly BD, Clarke M, McTigue O, Gervin M, Kamali M, et al. Incidence and clinical correlates of aggression and violence at presentation in patients with first episode psychosis. Schizophr Res 2005;72:161-8.

[13] Large M, Smith G, Nielssen O. The relationship between the rate of homicide by those with schizophrenia and the overall homicide rate: a systematic review and meta-analysis. Schizophr Res 2009;112:123-9.

[14] Lincoln TM, Hodgins S. Is lack of insight associated with physically aggressive behavior among people with schizophrenia living in the community? J Nerv Ment Dis 2008;196:62-6.

[15] Soyka M, Graz C, Bottlender R, Dirschedl P, Schoech H. Clinical correlates of later violence and criminal offences in schizophrenia. Schizophr Res 2007;94:89-98.

[16] Swanson JW, Swartz MS, Van Dorn RA, Elbogen EB, Wagner HR, Rosenheck RA, Stroup TS, McEvoy JP, Lieberman JA. A national study of violent behavior in persons with schizophrenia. Arch Gen Psychiatry 2006;63:490-9.

[17] Swartz MS, Swanson JW, Hiday VA, Borum R, Wagner HR, Burns BJ. Violence and severe mental illness: the effects of substance abuse and nonadherence to medication. Am J Psychiatry 1998;155:226-31.

[18] Waldheter EJ, Jones NT, Johnson ER, Penn DL. Utility of social cognition and insight in the prediction of inpatient violence among individuals with a severe mental illness. J Nerv Ment Dis 2005;193:609-18. 
[19] Yen CF, Yeh ML, Chen CS, Chung HH. Predictive value of insight for suicide, violence, hospitalization, and social adjustment for outpatients with schizophrenia: a prospective study. Compr Psychiatry 2002;43:443-7. 